\title{
The Quantitative Chemical Analysis of Early-type Stars
}

\author{
Lawrence H. Aller \\ The Observatory, University of Michigan, Ann Arbor, Michigan, U.S.A.
}

SuMmary

The University of Michigan programme for the determination of the abundances of the elements in hot stars involves an observational, a theoretical, and an experimental phase. A description of the spectrum of $\gamma$ Pegasi from $\lambda 3570-\lambda 3970$ and the profiles of the stronger lines in the photographic region illustrate the type of observational material that is being employed in this investigation. The point of view adopted for the theoretical programme is next described. Briefly, the model atmosphere of any given star must represent the observations of the continuous energy distribution, the Balmer discontinuity, the relative intensities of different spectral lines and the profiles of the stronger lines, notably those of hydrogen and helium. For this phase, a knowledge of the line-broadening coefficient is essential. Hence the emphasis on the experimental work with the luminous shock tube in which the Stark broadening of lines of hydrogen and other gases can be studied.

\section{INTRODUCTION}

THE spectra of the stars of early spectral class present some of the most engaging problems in astrophysics. Studies of stellar associations, such as the Lacerta aggregate, make it appear extremely likely that these stars are continuously being formed from the interstellar medium in the spiral arms of our galaxy (BLAAUw, 1952). The compositions of their atmospheres should reflect that of the interstellar medium, at least for young stars or for objects in which no mixing takes place between the atmosphere and interior.

Calculations of stellar models and evolutionary schemes, as well as questions concerning the origin of the elements, require an accurate analysis of the chemical compositions of these stars (see, e.g., ALLER, 1954). In order to calculate a stellar model, we must know the proportions of hydrogen, helium, carbon, nitrogen, and oxygen which are important in energy generation processes. These elements, as well as the heavier components of the stellar mixture, such as neon, magnesium, iron, etc., play a rôle in the determination of the opacity of the stellar interior. Improved data on nuclear cross-sections, as well as on the continuous absorption coefficients at high temperatures, require that we seek the most accurate knowledge of the abundances of the elements in the stars.

\section{The Observational Data}

At this point, let us turn to the observational data themselves to examine the nature of the material with which we have to work. The data are:

\section{(1) The Energy Distribution in the Continuous Spectrum}

The energy curves of early-type stars have been measured by a number of workers, among whom we may mention, especially, R. C. Williams (1938, 1939), Kienle and his associates (1940), and Barbier and Chalonge (1940), all of whom used photographic methods. More recently, CoDE and WHITford (1954) have applied photoelectric methods to this problem with great success. The accuracy of their observations presents a real challenge to the theory of model atmospheres. Measures 


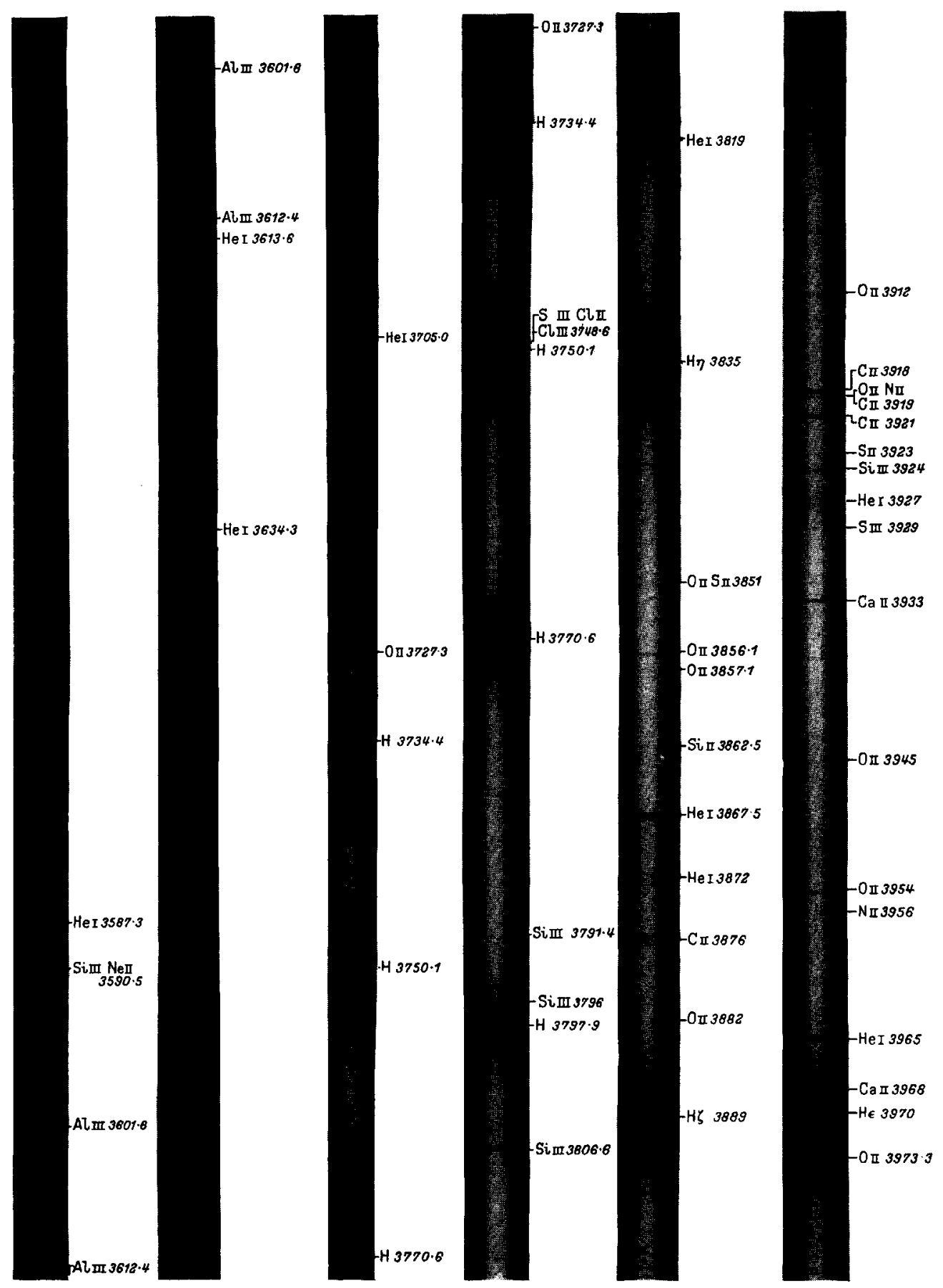

Fig. 1. The spectrum of $\gamma$ Pegasi; (I) $\lambda .3580 \AA-\lambda 3980 \AA$ 


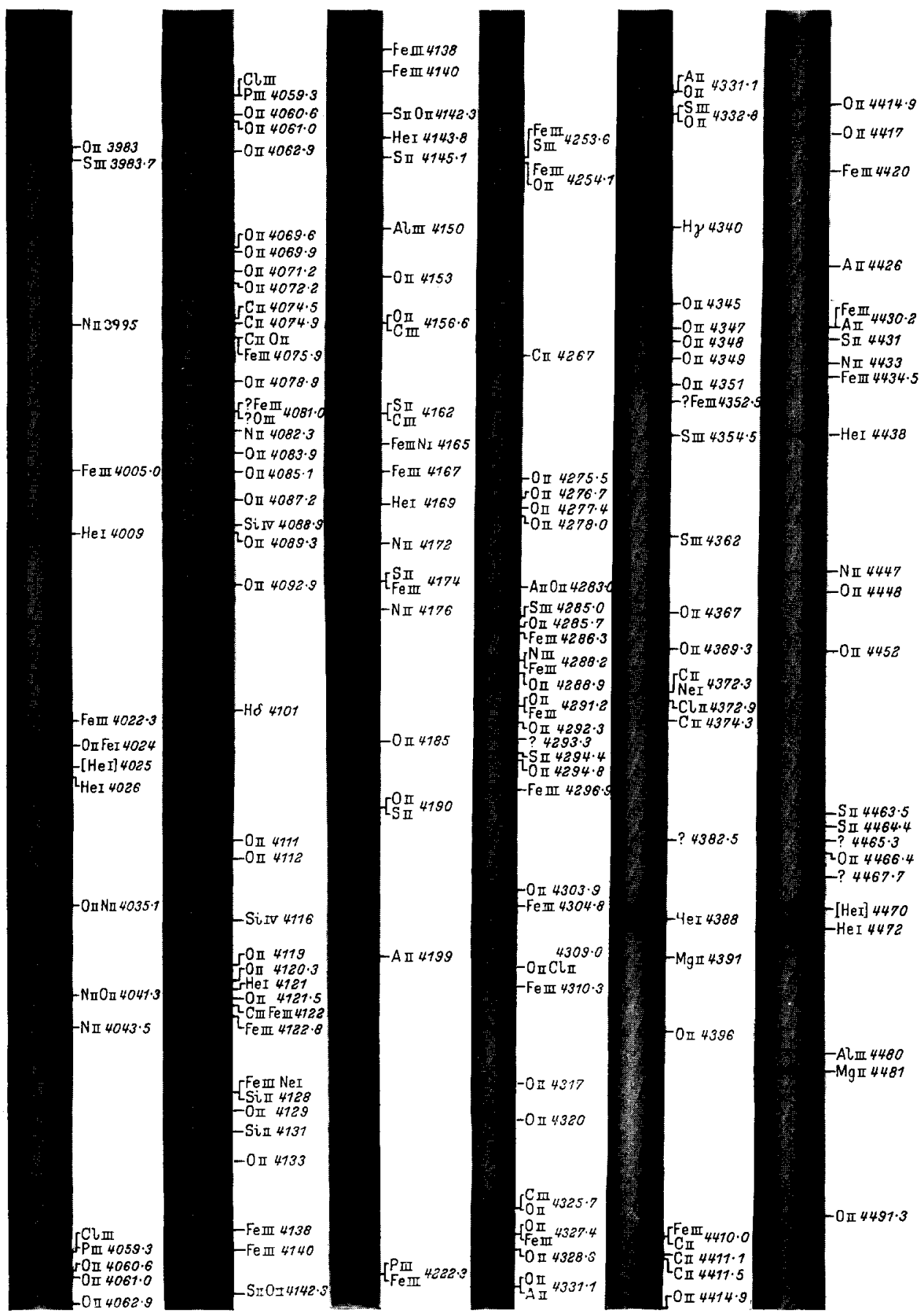

Fig. 2. The spectrum of, Pegasi; (II) $\lambda 3980 \AA-\lambda 4490 \AA$ 


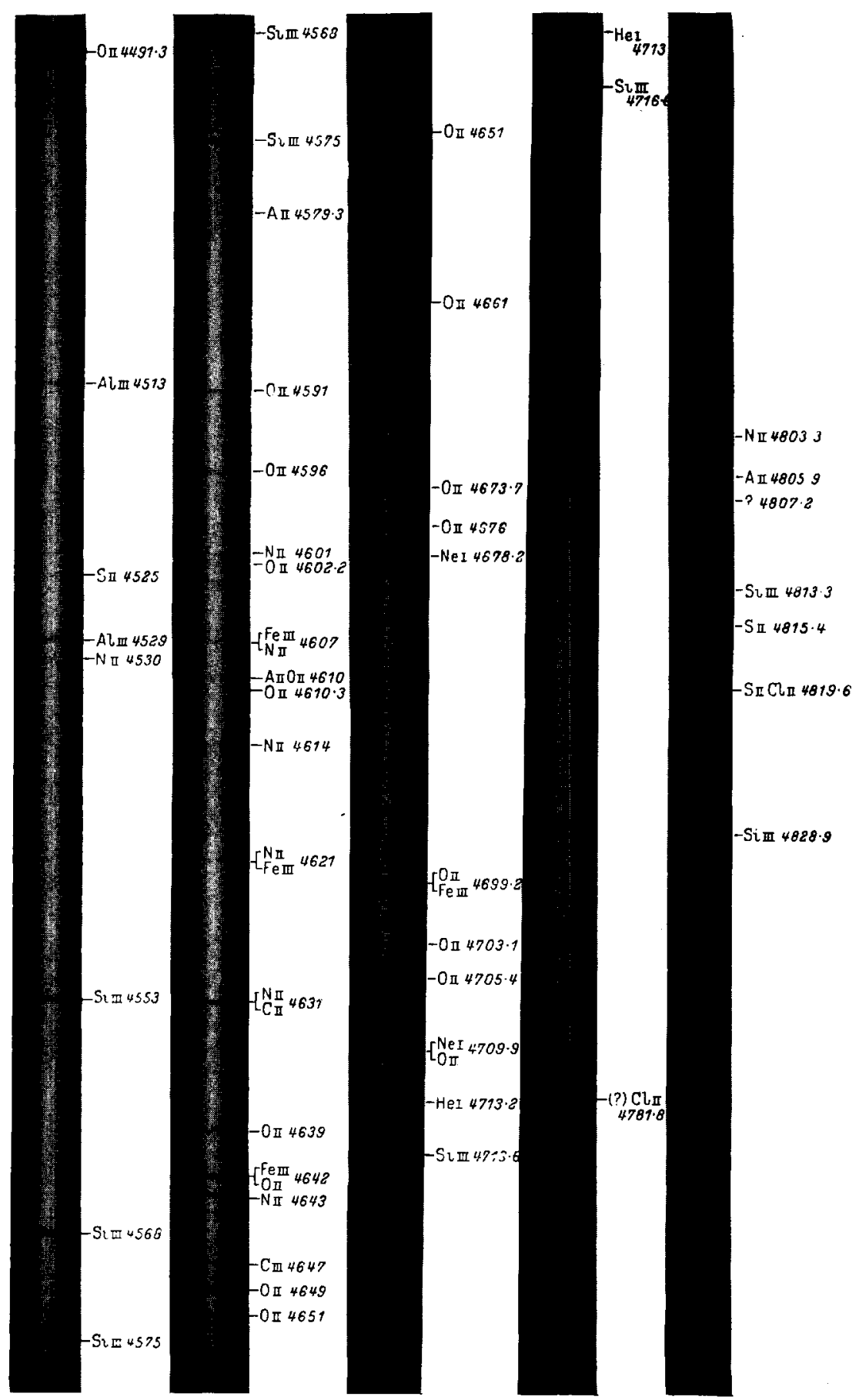

Fig. 3. The spectrum of $\gamma$ Pegasi; (III) $\lambda 4490 \AA-\lambda 4830 \AA$ 
of $I_{i}$ include, also, data on the Balmer discontinuity, I), whose numerical value depends on the effective temperature, surface gravity, and hydrogen/helium ratio. Although the observed energy distribution may be affected by space absorption, the Balmer discontinuity cannot be and, hence, serves as a useful quantity that must be accurately represented by the theory.

\section{(2) The Profiles and Equivalent Widths of Stellar Absorption Lines}

The shapes and total intensities of absorption lines may be measured upon high dispersion spectra. Although useful information on the profiles of strong diffuse lines, such as those of hydrogen, may be obtained from plates of lower dispersion, the detailed study of the $\mathrm{B}$ and $\mathrm{O}$ stars, with relatively sharp lines, requires dispersions at least as high as $10 \AA$ per mm.

Figs. 1, 2, and 3 show the spectrum of the bright B2.5 main sequence star, $\gamma$ Pegasi, as photographed at Mount Wilson. Notice the strong hydrogen lines which show sharp-cores except for the highest members of the series. The helium lines often also have broad wings with deep cores, although some, such as $\lambda 3927$, show very shallow profiles. The measured profiles of some of these lines are exhibited in Figs. 4 and 5 and may be compared with some of Miss UNDERHILL's results (1948a). In general, the equivalent widths of the weaker lines are comparable with the resolving power of the spectrograph so that meaningful profiles cannot be derived, and we must be content with equivalent widths.

The line identifications, marked on Figs 1, 2, and 3, are taken largely from the work of Miss Underfill (1948b) for $\lambda>3970$. Her identifications have been substantiated for nearly all of the stronger features by measurements of four or five of the best coudé plates. The weaker features are much more elusive. Many of the weak lines measured by Miss UnderhilL could not be seen on the Mount Wilson plates; on the other hand, I believe I have seen a fair number of weak lines not published in her list. Numerous faint lines have not been identified, while others arise from atoms whose abundance we would like to establish. Table 1 lists the lines measured upon four Mount Wilson coudé plates for the spectral region shortward of 23970. The first column gives the measured wavelength; the third column the quality of the identification, $A$ (certain), $B$ (very likely), $C$ (probable), and $D$ (poor). The fourth column gives the suggested identification with the multiplet number from Miss Moore's (1945) table. The second column, labelled "Intensity", requires some explanation. I decided to employ measured equivalent widths in units of milliAngstroms whenever possible. The stronger lines present no difficulty, but the weaker features are often badly confused by plate grain. In order to put these lines on the same scale as the stronger lines, I made the best estimate I could on the basis of the tracings. Nearly all lines weaker than $10 \mathrm{~m} \AA$ are marked with a $(:)$ which signifies that the intensities are rough estimates. A line listed as 5 :, for example, easily may have an equivalent width anywhere between 1 and 10 ! Yet, it has seemed better to distinguish between lines that scarcely seem to be detectable on tracings intensity $\sim 1$ or 2 and those that seem to be relatively well-established $(\sim 9$ or 10$)$. Our intensity scale, therefore, differs from conventional scales in its spread for the weaker lines which most writers might group together in classes called $(0)$ or $(00)$. I might have arbitrarily called all lines falling between 5 and $10(0)$ and those less than $5(00)$, with no loss in accuracy.

Measures of wavelengths and equivalent widths have been extended throughout 
Table 1. The spectrum of $\gamma$ Pegasi $(\lambda 3540-\lambda 3973)$

\begin{tabular}{|c|c|c|c|c|c|c|c|}
\hline Measured $\lambda$ & Intensity & $Q$ & Identification & Measured $\lambda$ & Intensity & $Q$ & Identification \\
\hline $3545 \cdot 92$ & 2: & $O$ & $3545 \cdot 84$ A II $(106)$ & $3635 \cdot 75$ & 一 & $D$ & $35 \cdot 67$ A II (68) \\
\hline \multirow{3}{*}{$3554 \cdot 22$} & 195 & $A$ & $54.39 \mathrm{He} \mathrm{I}(34)$ & $3637 \cdot 81$ & 2: & $C$ & 37.89 A II unclass. \\
\hline & & & $54 \cdot 39 \mathrm{Ne}$ II $(18)$ & $3638 \cdot 70$ & 5: & $C$ & $38 \cdot 70$ O III $(35)$ \\
\hline & & & $54.52 \mathrm{He}$ I & $3639 \cdot 75$ & - & $D$ & $39.85 \mathrm{~A}$ II + ? \\
\hline $3556 \cdot 56$ & 3: & $C$ & $56 \cdot 49$ P II (2l) & $3640 \cdot 40$ & 一 & - & 一 \\
\hline \multirow[t]{2}{*}{$3556 \cdot 8 \mathrm{I}$} & 10 & $D$ & $56 \cdot 91$ A II (29) & $3640 \cdot 73$ & $2:$ & $D$ & $40.89 \mathrm{~F}$ II $(11)+$ ? \\
\hline & & & 56.92 O III $(24)$ & $3642 \cdot 98$ & 3: & $D$ & $42 \cdot 80$ F II $(11)+$ ? \\
\hline 3561.05 & 2: & $C$ & $61 \cdot 04$ A II (106) & $3643 \cdot 82$ & 5: & $D$ & $43.89 \mathrm{Ne}$ II $(5)$ \\
\hline $3562 \cdot 09$ & 5 & $D$ & $62 \cdot 19$ A II $(106)+?$ & $3645 \cdot 79$ & 一 & - & 一 \\
\hline $3563 \cdot 02$ & - & $C$ & 62.95 He I $(33)$ & $3649 \cdot 80$ & 11 & - & $\longrightarrow$ \\
\hline $3565 \cdot 22$ & $2:$ & $C$ & $65 \cdot 02$ A II $(57)$ & $3650 \cdot 74$ & $\mathrm{p}$ & $D$ & $50 \cdot 70$ O III $(35)$ \\
\hline $3566 \cdot 54$ & $10:$ & $D$ & 66.43 P II $(22)+?$ & $3652 \cdot 10$ & 34 & $A$ & $51 \cdot 971$ \\
\hline $3570 \cdot 41$ & 9 & $D$ & $70 \cdot 34 \mathrm{P}_{\text {II }}(18)$ & & & & $52 \cdot 119 \mathrm{He}$ I $(27)$ \\
\hline $3570 \cdot 76$ & 1: & $D$ & $70.77 \mathrm{~A}$ II $(69)$ & $3652 \cdot 67$ & 2: & $B$ & $52.65 \mathrm{Fe}$ III \\
\hline $3572 \cdot 53$ & 5: & $O$ & $72 \cdot 64$ Fe III $(105)$ & $3652 \cdot 95$ & - & $D$ & $53 \cdot 00$ O III $(35)$ \\
\hline $3577 \cdot 35$ & - & - & - & $3653 \cdot 45$ & $\cdots$ & - & - \\
\hline $3581 \cdot 55$ & 3: & $C$ & $81 \cdot 62 \mathrm{~A}$ II $(56)$ & $3655 \cdot 29$ & 4 & $C$ & $55 \cdot 29 \mathrm{~A}$ II $(82)$ \\
\hline $3582 \cdot 41$ & $6:$ & $C$ & $82 \cdot 35 \mathrm{~A}$ II $(56)$ & $3656 \cdot 68$ & $3:$ & $D$ & $56 \cdot 67 \mathrm{H}$ \\
\hline $3584 \cdot 93$ & 3: & $C$ & $84.98 \mathrm{C}$ Ir $(23)$ & & & & $56 \cdot 61 \mathrm{~S}$ III $(6)$ \\
\hline \multirow[t]{2}{*}{$3586 \cdot 07$} & 14 & $B$ & $86 \cdot 12 \mathrm{Fe}$ III $(36)$ & $3657 \cdot 29$ & $2:$ & $D$ & $57.269 \mathrm{H}$ \\
\hline & & & $85 \cdot 83$ C II (23) & $3657 \cdot 91$ & 6: & $D$ & $57.926 \mathrm{H}$ \\
\hline \multirow[t]{2}{*}{$3587 \cdot 29$} & 290 & $A$ & $87 \cdot 252$ & $3658 \cdot 80$ & - & $D$ & $58.641 \mathrm{H}$ ? \\
\hline & & & $87 \cdot 396$ He I (31) & $3659 \cdot 45$ & $2:$ & $D$ & $59.423 \mathrm{H}$ \\
\hline $3588 \cdot 36$ & $\mathbf{p}$ & $C$ & $88 \cdot 44$ A II $(56)$ & $3659 \cdot 79$ & 3 . & $D$ & $59 \cdot 84 \mathrm{Cl}$ II $(7)$ \\
\hline $3589 \cdot 03$ & 1: & $D$ & 88.92 C II $(23)$ & $3660 \cdot 00$ & $3:$ & $D$ & $59 \cdot 93 \mathrm{Ne}$ II $(33)$ \\
\hline $3589 \cdot 87$ & 9 & $D$ & $89.77 \mathrm{Fe}$ III & $3660 \cdot 24$ & 一. & $D$ & $60 \cdot 279 \mathrm{H}$ \\
\hline \multirow[t]{2}{*}{$3590 \cdot 52$} & 19 & $B$ & $90 \cdot 46$ Si III (7) & $3660 \cdot 44$ & 3: & $D$ & $60 \cdot 44$ A II $(116)$ \\
\hline & & & $90 \cdot 47 \mathrm{Ne}$ II $(32)$ & $3660 \cdot 85$ & $5:$ & - & $60 \cdot 85 \mathrm{Fe}$ III $(93)$ \\
\hline \multirow[t]{2}{*}{$3593 \cdot 68$} & - & $D$ & $93 \cdot 60 \mathrm{~N}$ II $(26)$ & $3661 \cdot 32$ & 一 & $D$ & $61 \cdot 22 \mathrm{H}$ \\
\hline & & & 93.76 A II (117) & $3662 \cdot 41$ & 一 & $E$ & $62 \cdot 26 \mathrm{H}$ ? \\
\hline $3594 \cdot 27$ & 2: & $D$ & $94 \cdot 18 \mathrm{Ne}$ II $(34)$ & $3663 \cdot 70$ & $3: \mathrm{d}$ & $D$ & 63.47 S II $(16)+$ \\
\hline \multirow[t]{2}{*}{$3594 \cdot 52$} & 3: & $C$ & $94 \cdot 41$ A II $(23)$ & & & & $63.98 \mathrm{Fe}$ III (24) \\
\hline & & & $94 \cdot 46 \mathrm{~S}$ II $(16)$ & $3664 \cdot 18$ & 2: & $D$ & $64.09 \mathrm{Ne}$ II $(\mathrm{l})$ \\
\hline $3595 \cdot 96$ & 4: & $B$ & $95.99 \mathrm{~S}$ II $(4)$ & & & & $64 \cdot 20$ P II $(18)$ \\
\hline \multirow[t]{2}{*}{$3599 \cdot 21$} & 13 & $B$ & $99 \cdot 304$ & $3664 \cdot 65$ & 4: & $C$ & $64.679 \mathrm{H}$ \\
\hline & & & $99.442 \mathrm{He} \mathrm{I}$ & $3669 \cdot 07$ & 16 & $\mathrm{C}$ & $69.05 \mathrm{~S}$ II $(16)$ \\
\hline $3600 \cdot 19$ & 1: & $D$ & $00 \cdot 22$ A II (115) & $3670 \cdot 37$ & $5:$ & $C$ & $70 \cdot 28 \mathrm{Cl}$ III $(1)$ \\
\hline $3600 \cdot 91$ & 14 & $B$ & $00.93 \mathrm{Fe}$ III $(36)$ & $3676 \cdot 02$ & - & $\vec{D}$ & $\overline{-7}=0$ \\
\hline \multirow[t]{2}{*}{$3601 \cdot 64$} & 40 & $A$ & $01.62 \mathrm{Al}$ III (1) & $3676 \cdot 28$ & 一 & $D$ & $76 \cdot 27$ P II $(19)$ \\
\hline & & & $01.51 \mathrm{~A}+1(4)$ & 3676.42 & - & - & $76 \cdot 365 \mathrm{H}$ \\
\hline $3602 \cdot 20$ & 2: & $D$ & $02 \cdot 10 \mathrm{Cl}$ III (1) & $\mathbf{3 6 7 6 . 5 8}$ & 一 & 一 & - \\
\hline $3603 \cdot 44$ & $\longrightarrow$ & $C$ & $03 \cdot 46 \mathrm{~A}$ II $(57) ?$ & $3680 \cdot 11$ & 4: & $D$ & $80.06 \mathrm{~A}$ II $(115)$ \\
\hline \multirow[t]{2}{*}{$3603 \cdot 90$} & 9: & $B$ & $03.88 \mathrm{Fe}$ III (36) & $3682 \cdot 34$ & 4: & $D$ & $82 \cdot 56$ A II $(29)$ \\
\hline & & & 03.91 A II $(43,68)$ & & & & $82.05 \mathrm{Cl}$ III (1) \\
\hline $3604 \cdot 53$ & 3: & $D$ & $04.51 \mathrm{Cl}$ II $(78)$ & $3683 \cdot 31$ & 5: & $D$ & $83.39 \mathrm{Cl}$ III (12) ? \\
\hline $3605 \cdot 75$ & $\ldots$ & $C$ & 05.89 A II $(30)+$ ? & $3684 \cdot 53$ & - & - & - \\
\hline $3607 \cdot 81$ & - & - & - & $3690 \cdot 44$ & $3:$ & $D$ & $90 \cdot 60 \mathrm{Fe}$ III $(85)+$ ? \\
\hline $3609 \cdot 08$ & $2:$ & $C$ & $09.09 \mathrm{~N}$ II (26) & $3692 \cdot 37$ & $9:$ & $D$ & $92 \cdot 33$ A II (4) \\
\hline $3609 \cdot 64$ & 1: & $C^{\prime}$ & $09 \cdot 61$ C III (10)? & $3694 \cdot 13$ & 2: & $\bar{D}$ & $94.22 \mathrm{Ne}$ II (1) \\
\hline $3610 \cdot 49$ & - & 一 & - & $3697 \cdot 25$ & 140 & $O$ & $97 \cdot 154 \mathrm{H}$ \\
\hline $3611 \cdot 72$ & $6:$ & $B$ & $11 \cdot 72 \mathrm{Fe}$ III (36) & $3700 \cdot 82$ & - & - & - \\
\hline \multirow[t]{2}{*}{$3612 \cdot 38$} & 25 & $A$ & $12 \cdot 352$ Al III (1) & $3701 \cdot 35$ & - & - & $一$ \\
\hline & & & $12 \cdot 35$ Ne II $(26)$ & $3702 \cdot 01$ & $3:$ & $C$ & $02 \cdot 09 \mathrm{Al}$ III (4) \\
\hline $3613 \cdot 10$ & 7: & $D$ & $13 \cdot 03 \mathrm{~S}$ II $(4)$ & $3702 \cdot 95$ & - & - & - \\
\hline $3613 \cdot 63$ & 89 & $A$ & $13 \cdot 641 \mathrm{He} \mathrm{I}$ & $3703 \cdot 67$ & 520 & $D$ & $03.855 \mathrm{H}$ \\
\hline $3615 \cdot 40$ & 4: & 一 & $\rightarrow$ & $3705 \cdot 05$ & 300 & $A$ & $05.003 \mathrm{He} \mathrm{I}$ \\
\hline 3615.69 & 1: & $C$ & $15 \cdot 64 \mathrm{Mg}$ II & & & & $05 \cdot 140 \mathrm{He} \mathrm{I}$ \\
\hline $3615 \cdot 88$ & 2: & $C$ & $15.88 \mathrm{~N}$ II $(26)$ & $3705 \cdot 98$ & - & $D$ & 06.06 P II (20)? \\
\hline $3619 \cdot 18$ & - & 一 & $\ldots$ & $3706 \cdot 65$ & - & - & \\
\hline $3619 \cdot 66$ & - & - & - & $3707 \cdot 04$ & - & $D$ & $06.94 \mathrm{~A}$ II \\
\hline $3621 \cdot 05$ & 4: & $C$ & $21 \cdot 06$ A II $(4)$ & $3707 \cdot 29$ & 4: & $D$ & $07 \cdot 24 \mathrm{O}$ III $(14)$ \\
\hline $3626 \cdot 46$ & - & $D$ & 26.53 S III & & & & $07 \cdot 34 \mathrm{Cl}$ III (9) \\
\hline $3628 \cdot 06$ & 4: & $D$ & $28 \cdot 06 \mathrm{Ne}$ II (41) & $3709 \cdot 33$ & 5 & $B$ & $09 \cdot 37 \mathrm{~S}$ III (1) \\
\hline $3631 \cdot 27$ & 8 & $D$ & $31 \cdot 27 \mathrm{Ne}$ II (2) & $3711 \cdot 95$ & 580 & $A$ & $11.973 \mathrm{H}$ \\
\hline $3632 \cdot 04$ & 22 & $B$ & $32 \cdot 02 \mathrm{~S}$ III (1) & $3712 \cdot 78$ & 5: & $C$ & $12 \cdot 75 \mathrm{O}$ II (3) \\
\hline $3634 \cdot 27$ & 400 & $A$ & $\begin{array}{l}34 \cdot 235 \mathrm{He} \text { I } \\
34 \cdot 373 \mathrm{He} \text { I }\end{array}$ & & & & \\
\hline
\end{tabular}


Trable 1-contd.

\begin{tabular}{|c|c|c|c|c|c|c|c|}
\hline Measured $\lambda$ & Intensity & $Q$ & Identification & Measured $\lambda$ & Intensity & $Q$ & Identification \\
\hline & & & & & & - & $\cdots .$. \\
\hline \multirow[t]{3}{*}{$3713 \cdot 16$} & 3: & $(!$ & I3.103 Al пा (4) & $3786 \cdot 81$ & 4: & C & $86 \cdot 70 \mathrm{P}$ I. (1) \\
\hline & & & $13.09 \mathrm{Ne}$ II $(5)$ & & & & $86.94 \mathrm{Fe}$ III (7]) \\
\hline & & & $13.03 \mathrm{~A}$ пі (114) & $3788 \cdot 14$ & $\cdots$ & $\cdots$ & - \\
\hline $3713 \cdot 94$ & - & $O$ & 14.030 III (14) & $3789 \cdot 00$ & 4 & () & $88.91 \mathrm{Fe} 1 \mathrm{II}(102)$ \\
\hline $3715 \cdot 15$ & $\tilde{5}:$ & $(!$ & $15.08 O_{111}(14)$ & 3790.98 & 1: & l) & 90.96 Ne II $(30)$ \\
\hline $3716 \cdot 39$ & $2:$ & - & - & $3791 \cdot 42$ & 30 & $A$ & 91.41 Si $[11(5)$ \\
\hline \multirow[t]{2}{*}{$3717 \cdot 72$} & 11 & $C !$ & 17.776 S III (6) & $3793 \cdot 16$ & 2: & -- & - \\
\hline & & & $17.63 \mathrm{P}$ III $(10)$ & $3793 \cdot 52$ & 2 & $(!$ & $93.52 \mathrm{Fe} 111(7 \mathrm{~L})$ \\
\hline $3721 \cdot 89$ & 1070 & $A$ & $21 \cdot 940 \mathrm{H}$ & & & & $93 \cdot 61$ J' पा (1) \\
\hline $3726 \cdot 23$ & - & - & - & 3793.98 & - & .. & - \\
\hline $3726 \cdot 64$ & - & - & - & $3795 \cdot 35$ & $2:$ & ( & $95 \cdot 37$ A III (5) \\
\hline $3727 \cdot 33$ & 39 & $A$ & $27 \cdot 330$ и (3) & $3796 \cdot 10$ & 12 & $A$ & $9(3.11$ Si $5(5)$ \\
\hline $3727 \cdot 76$ & 12 & $\ldots$ & - & $3797 \cdot 92$ & 4280 & $A$ & $97.90 \mathrm{H}$ \\
\hline \multirow[t]{2}{*}{$3728 \cdot 55$} & $6:$ & l) & 28.490 III $(30)$ & $3801 \cdot 12$ & $\ldots$ & - & - \\
\hline & & & $28 \cdot 67$ P II $(22)$ & $3801 \cdot 65$ & --- & --- & - \\
\hline \multirow[t]{2}{*}{$3729 \cdot 35$} & 8 & $B$ & $29 \cdot 340$ І $(62)$ & $3805 \cdot 23$ & $3:$ & () & $05 \cdot 24(7)$ + $(62)$ \\
\hline & & & $29 \cdot 29$ A II $(10)$ & $3805 \cdot 78$ & 52 & $(?$ & $05.765 \mathrm{He} \mathrm{I}(63)$ \\
\hline $3729 \cdot 76$ & - & I) & $29 \cdot 70 O_{\text {III }}(30)$ & $3806 \cdot 52$ & 99 & $A$ & 06.56 Si III $(5)$ \\
\hline $3730 \cdot 35$ & $\ldots$ & - & -- & $3809 \cdot 69$ & $\ldots$ & $(?$ & 09.67 S I $(50)$ \\
\hline $3732 \cdot 00$ & - & I) & $32 \cdot 130$ III $(14)$ & $3810 \cdot 02$ & 4: & $(!$ & $10 \cdot 1001$ [1 $(62)$ \\
\hline \multirow[t]{2}{*}{$3732 \cdot 98$} & 28 & $A$ & $32 \cdot 861$ & $3811 \cdot 7 \mathrm{I}$ & 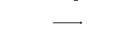 & $\ldots$ & $11.80 \mathrm{~s}$. \\
\hline & & & $32 \cdot 992$ He I & $3812 \cdot 15$ & - & & $\ldots \ldots$ \\
\hline $3734 \cdot 41$ & 1770 & $A$ & $34.370 \mathrm{H}$ & $3819 \cdot 6.5$ & 1290 & $A$ & $19 \cdot 606$ \\
\hline $3738 \cdot 40$ & l: & - & $\ldots$ & & & & $19.761 \mathrm{He}$ I \\
\hline $3738 \cdot 84$ & - & - & -- & $3825 \cdot 70$ & 5 & $B$ & $25 \cdot 70 \mathrm{~A}$ I $(129)$ \\
\hline $3739 \cdot 33$ & 1: & $D$ & $39 \cdot 13 \mathrm{~K}$ II (I) - ! & $3831 \cdot 37$ & 4 & $C^{\prime}$ & $31 \cdot 41 \mathrm{~S} \mathrm{II}$ \\
\hline $3739 \cdot 76$ & $7:$ & 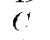 & $39 \cdot 920_{\text {II }}(31)$ & $3831 \cdot 74$ & $2:$ & $C$ & $31.75 \mathrm{Fe}$ III $(109)$ \\
\hline $3743 \cdot 45$ & 3: & $C$ & $43 \cdot 40 \mathrm{Fe}$ III & & & & 31.85 S III $(5)$ \\
\hline $3744 \cdot 23$ & $2:$ & $B$ & $44 \cdot 22$ P III $(10)$ & $3833 \cdot 10$ & 6 & $(!$ & $33 \cdot 10 O_{11}(13)$ \\
\hline $3745 \cdot 52$ & - & $\ldots$ & - & $3835 \cdot 41$ & 4640 & $A$ & $35 \cdot 386 \mathrm{H}$ \\
\hline $3745 \cdot 81$ & - & $(!$ & $45.83 \mathrm{~N}$ 11I (4) & $3838 \cdot 36$ & 1: & $R$ & $38.316 \mathrm{~S}$ III $(5)$ \\
\hline $3746 \cdot 4=$ & - & $(!$ & $46.46 \mathrm{~A}$ II $(130)$ & & & & $38 \cdot 37 \mathrm{Cl}$ IL $(69)$ \\
\hline $3746 \cdot 83$ & 一 & $D$ & 46.92 A II $(67)$ & & & & $38.39 \mathrm{~N}$ II $(30)$ \\
\hline \multirow[t]{3}{*}{$3748 \cdot 65$} & - & I) & $48.73 \mathrm{~S}$ III & $3840 \cdot 44$ & - & - & - \\
\hline & & & $46 \cdot 46 \mathrm{Cl}$ II $(6)$ & $3844 \cdot 75$ & $3:$ & 1) & $44 \cdot 75$ A II $(54)$ \\
\hline & & & $48 \cdot 81$ Cl III $(5)$ & $3845 \cdot 70$ & $4:$ & $\theta$ & $45.68 \mathrm{Fe} 111(35)$ \\
\hline $3750 \cdot 29$ & 2840 & $A$ & $50 \cdot 154 \mathrm{H}$ & & & & $45 \cdot 69$ \\
\hline $3752 \cdot 35$ & - & 一 & - & & & & $45 \cdot 84)^{\mathrm{Cl}} 1 \mathrm{Il}(26)$ \\
\hline $3753 \cdot 47$ & l: & 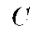 & $53 \cdot 53 \mathrm{~A}_{\text {II }}(80,128)$ & $3846 \cdot 47$ & - & $\cdots \cdots$ & - \\
\hline $3754 \cdot 02$ & - & $c$ & $54 \cdot 06 \mathrm{~A}$ II $(115)$ & $3847 \cdot 44$ & 3: & l) & $47 \cdot 38 \mathrm{~N}$ II $(30)$ \\
\hline \multirow[t]{2}{*}{$3754 \cdot 65$} & - & 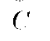 & $54.62 \mathrm{~N}$ III (4) & $3847 \cdot 91$ & 9 & $C^{\prime}$ & 47.890 II $(12)$ \\
\hline & 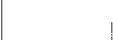 & & 54.67 O III $(2)$ & $3848 \cdot 26$ & $1 \ddot{3}$ & (! & $48 \cdot 24 \mathrm{Mg}$ il $(5)$ \\
\hline $3755 \cdot 12$ & 一 & - & - & $3848 \cdot 61$ & - & $-\cdots$ & -- \\
\hline $3756 \cdot 38$ & - & I) & $56 \cdot 10 \mathrm{He}_{1}(66)$ & $3848 \cdot 97$ & - & -- & - \\
\hline $3756 \cdot 78$ & - & - & - & $3849 \cdot 91$ & $t i$ & $B$ & $49 \cdot 99 \mathrm{~F}$ II $(1)$ \\
\hline $3757 \cdot 13$ & 7 & C & $57 \cdot 210$ III $(2)$ & $3850 \cdot 30$ & $7:$ & $(!$ & $50.40 \mathrm{Mg}$ it (5) \\
\hline $3757 \cdot 60$ & - & D) & $57.60 \mathrm{~N}$ III (li) & $385 \mathrm{l} \cdot 00$ & 28 & $B$ & 51.04 O II (12) \\
\hline $3758 \cdot 69$ & - & $\theta$ & $57 \cdot 66 \mathrm{~N}$ III (II) & & & & 50.93 S II $(50)$ \\
\hline $3759 \cdot 84$ & $6:$ & $\theta$ & $59 \cdot 87$ O III (2) & & & & $50 \cdot 97 \mathrm{Cl} 11(25)$ \\
\hline $3760 \cdot 11$ & - & - & - & $3851 \cdot 47$ & 7 & ( & 51.470 II (13) \\
\hline \multirow[t]{2}{*}{$3762 \cdot 47$} & 8 & 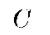 & $62 \cdot 41$ Si IV (3) & & & & $51 \cdot 38 \mathrm{Cl}$ II $(25)$ \\
\hline & & & $62 \cdot 62 \mathrm{~N}$ III (11) & $3852 \cdot 40$ & - & - & \\
\hline $3762 \cdot 80$ & 1: & I) & $62.63011(31)-+?$ & $3853 \cdot 66$ & 18 & $A$ & $53 \cdot 657$ Si I (1) \\
\hline $3770 \cdot 66$ & 3600 & $A$ & $70 \cdot 632 \mathrm{H}$ & $3854 \cdot 36$ & 1: & - & - \\
\hline $3774 \cdot 28$ & $4:$ & $D$ & $74 \cdot 25$ Cl I1 (6) & $3855 \cdot 09$ & 13 & $B$ & $55.08 \mathrm{~N}_{15}(30)$ \\
\hline 3774.98 & 6 & $\theta$ & $75.03 \mathrm{P}_{\text {II }}(19)$ & & & & $55 \cdot 18$ A II $(81)$ \\
\hline \multirow[t]{3}{*}{3777.41} & 6 & $C$ & $77 \cdot 43$ Fe III $(95)$ & $3856 \cdot 02$ & 59 & $A$ & $56 \cdot 02 \mathrm{Si}$ II $(1)$ \\
\hline & & & $77.55 \mathrm{~A}$ II $(81)$ & & & & $56 \cdot 07 \mathrm{~N}$ II $(30)$ \\
\hline & & & $77.600 \mathrm{II}(3 \mathrm{I})$ & & & & $56 \cdot 16 \mathrm{O}_{\text {II }}(12)$ \\
\hline 3778.90 & $7:$ & $B$ & $78.90 \mathrm{~S}$ III $(5)$ & $3857 \cdot 15$ & 14 & $A$ & $57 \cdot 180$ II $(13)$ \\
\hline & $6:$ & ( & 82.6 S II $(23)$ & $3857 \cdot 67$ & -.. & - & — \\
\hline \multirow[t]{2}{*}{$3783 \cdot 24$} & 10 & l) & $83 \cdot 165$ S II (41) & $3859 \cdot 30$ & 5 & c & $59 \cdot 26 \mathrm{~S}$ II $(30)$ \\
\hline & & & $83 \cdot 19 \mathrm{~K}$ II (2) & $3860 \cdot 12$ & 7 & i) & $60 \cdot 15$ S II (41) \\
\hline 3784.82 & 8 & $C^{\prime}$ & $84.886 \mathrm{He}$ I $(64)$ & $3860 \cdot 65$ & 9 & $B$ & $60 \cdot 64$ S II $(50)$ \\
\hline & & & & & & & $60 \cdot 64 \mathrm{~S}$ III $(5)$ \\
\hline $3785 \cdot 64$ & 一 & 一 & $\ldots$ & $3861 \cdot 03$ & 8 & 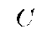 & $60 \cdot 98 \mathrm{Cl}$ II $(25)$ \\
\hline $3786 \cdot 40$ & 5: & $B$ & $86 \cdot 40 \mathrm{~A}$ II $(3)$ & $3861 \cdot 41$ & $3:$ & $C^{\prime}$ & $61 \cdot 40$ Cl ir (25) \\
\hline
\end{tabular}


Table 1-contd.

\begin{tabular}{|c|c|c|c|c|c|c|c|}
\hline Measured $\lambda$ & Intensity & $Q$ & Identification & Measured $\lambda$ & Intensity & $Q$ & Identiflcation \\
\hline $3861 \cdot 89$ & 1: & $C$ & $61 \cdot 95 \mathrm{Cl}$ II $(84)$ & $3919 \cdot 30$ & 36 & $A$ & $19 \cdot 287$ OII $(17)$ \\
\hline $3862 \cdot 60$ & 34 & $A$ & $62 \cdot 592 \mathrm{Si}$ II (1) & $3919 \cdot 75$ & - & - & - \\
\hline 3863.53 & 15 & $A$ & $63.50 \mathrm{O}$ II $(12)$ & $3920 \cdot 65$ & 93 & $\boldsymbol{A}$ & $20 \cdot 677$ C II (4) \\
\hline $3864 \cdot 04$ & 9 & $C$ & $64 \cdot 130$ II $(11)$ & $3921 \cdot 20$ & - & - & - \\
\hline $3864 \cdot 47$ & 20 & $B$ & 64.45 O II $(12)$ & $3922 \cdot 72$ & 1: & $D$ & $22 \cdot 72$ P III (9) \\
\hline $3864 \cdot 65$ & 12 & $B$ & $64 \cdot 60$ Cl II (12) & & & & $22 \cdot 63 \mathrm{~S}$ II $(60)$ \\
\hline & & & $64 \cdot 68$ O II (12) & $3923 \cdot 43$ & 19 & $A$ & $23.483 \mathrm{~S}$ II $(55)$ \\
\hline $3867 \cdot 52$ & 193 & $A$ & $\begin{array}{l}67 \cdot 477 \\
67 \cdot 631 \mathrm{He} \mathrm{I}\end{array}$ & $\begin{array}{l}3924 \cdot 46 \\
3925 \cdot 32\end{array}$ & 43 & $\underline{A}$ & $24 \cdot 44 \mathrm{Si} I I I$ \\
\hline $3868 \cdot 53$ & 6: & $B$ & 68.53 A II $(90)$ & $3925 \cdot 65$ & - & $C$ & $25.71 \mathrm{~A}$ II $(105)$ \\
\hline 3868.90 & 6: & $C$ & 68.84 C It (18) & $3926 \cdot 65$ & 570 & $A$ & $26.530 \mathrm{He} \mathrm{I}$ \\
\hline $3871 \cdot 76$ & 2: & $C$ & $71 \cdot 62$ C II $(18)$ & $3927 \cdot 62$ & - & - & - \\
\hline $3871 \cdot 9$ & 364 & $D$ & $71 \cdot 82 \mathrm{He} \mathrm{I}$ & 3927.92 & - & - & - \\
\hline $3872 \cdot 34$ & $\begin{array}{l}1: \\
1:\end{array}$ & $D$ & $\begin{array}{l}72 \cdot 15 \mathrm{~A}_{\text {II }} \\
72 \cdot 45 \mathrm{O}_{\text {II }}\end{array}$ & $3928 \cdot 60$ & 22 & $A$ & $\begin{array}{l}28 \cdot 615 \text { S III } \\
28 \cdot 62 \mathrm{~A} \text { II }(10)\end{array}$ \\
\hline $3874 \cdot 42$ & $6:$ & - & $\longrightarrow$ & $3934 \cdot 45$ & 2: & $C$ & $34 \cdot 41 \mathrm{~N}$ III $(8)$ \\
\hline $3875 \cdot 25$ & 8 & $C$ & $75 \cdot 26 \mathrm{~A}$ II $(2)$ & $3936 \cdot 04$ & 32 & 一 & - \\
\hline $3876 \cdot 13$ & 55 & $A$ & $76 \cdot 051$ & $3938 \cdot 43$ & 6 & $D$ & $38.52 \mathrm{~N}$ III (8) \\
\hline & & & $76 \cdot 188 \mathrm{C}$ II $(33)$ & $3939 \cdot 48$ & 6 & $C$ & $39 \cdot 49 \mathrm{~S}$ II $(45)$ \\
\hline $3876 \cdot 41$ & 35 & $A$ & $76 \cdot 409 \mathrm{C}$ II $(33)$ & $3944 \cdot 32$ & 7 & $C$ & $44 \cdot 27$ A II $(2)$ \\
\hline $3876 \cdot 67$ & 27 & $A$ & $76 \cdot 670$ C II (33) & $3945 \cdot 04$ & 40 & $A$ & $45 \cdot 048$ O II $(6)$ \\
\hline $3878 \cdot 18$ & 20 & $C$ & $\begin{array}{l}78 \cdot 180 \mathrm{He} \mathrm{I} \\
78 \cdot 22 \mathrm{C} \text { II }(33)\end{array}$ & & & & $\begin{array}{l}45.06 \mathrm{~S} \text { II }(33) \\
45.08 \mathrm{Fe} \text { III }(69)\end{array}$ \\
\hline $3879 \cdot 59$ & 7 & $B$ & $79 \cdot 60$ C II $(33)$ & & & & $45 \cdot 10$ C II $(32)$ \\
\hline $3880 \cdot 59$ & 8 & $A$ & $80 \cdot 59$ C II (33) & $3946 \cdot 98$ & 7 & $C$ & $46 \cdot 98 \mathrm{~S}$ II $(45)$ \\
\hline $3882 \cdot 19$ & 25 & $A$ & $82 \cdot 197$ O II (12) & & & & $47 \cdot 10$ Fe III $(23,69)$ \\
\hline $3883 \cdot 09$ & 4: & $C$ & $83 \cdot 15011(12)$ & $3948 \cdot 25$ & 6 & $D$ & $48 \cdot 15 \mathrm{C}_{\text {II }}(32)+$ \\
\hline $3885 \cdot 93$ & $3:$ & $C$ & 85.99 C III (15) & 3948.98 & $2:$ & $D$ & $48 \cdot 979 \mathrm{~A} I(2)$ \\
\hline $3889 \cdot 04$ & 4710 & $A$ & $89 \cdot 051 \mathrm{H}$ & $3949 \cdot 81$ & 1: & $D$ & $49 \cdot 45$ C II $(31)$ \\
\hline $3893 \cdot 34$ & 4: & $D$ & $93 \cdot 14$ A II (9l) & & 7: & $D$ & 49.96 Cl ir $(36)$ \\
\hline $3894 \cdot 88$ & 2: & $D$ & $95 \cdot 03$ P III (9) & $3950 \cdot 35$ & 6 & $C$ & 50.42 S II $(45)$ \\
\hline $3895 \cdot 28$ & 4: & $O$ & $95 \cdot 26 \mathrm{~A}$ II $(55)$ & $3952 \cdot 07$ & 12 & $C$ & $52 \cdot 08 \mathrm{C}_{\text {II }}(32)$ \\
\hline $3905 \cdot 93$ & - & $\longrightarrow$ & - & $3953 \cdot 76$ & 6 & $B$ & $53 \cdot 76 \mathrm{Fe}$ III $(69)$ \\
\hline $3906 \cdot 93$ & - & $O$ & 06.95 S II (3) & $3954 \cdot 36$ & 44 & $A$ & $54 \cdot 372$ O II (6) \\
\hline $3907 \cdot 44$ & 11 & $C$ & $07 \cdot 45$ O II (1I) & & & & $54 \cdot 38 \mathrm{Fe}$ III $(120)$ \\
\hline $3909 \cdot 34$ & - & - & - & $3955 \cdot 18$ & 一 & 一 & 一 \\
\hline $3911 \cdot 43$ & 3: & $D$ & $11.58 \mathrm{~A}$ II $(54)$ & $3955 \cdot 85$ & 25 & $A$ & $55 \cdot 851 \mathrm{~N}$ II $(6)$ \\
\hline & & & $11 \cdot 32 \mathrm{~S}$ II & $3956 \cdot 82$ & - & $\bar{T}$ & - $\ldots$ \\
\hline $3911 \cdot 97$ & 50 & $A$ & $\left.\begin{array}{l}11 \cdot 960 \\
12 \cdot 088\end{array}\right\}$ O II (17) & $3961 \cdot 54$ & - & $D$ & $\begin{array}{l}61.55 \text { S III (8) } \\
61.59 \text { O III (17) }\end{array}$ \\
\hline $3912 \cdot 64$ & - & - & - & $3962 \cdot 61$ & - & - & — \\
\hline $3913 \cdot 81$ & 14: & $C$ & $13.92 \mathrm{Cl} \mathrm{II}(68)+$ ? & $3964 \cdot 72$ & 159 & $A$ & $64.727 \mathrm{He} \mathrm{I} \mathrm{(5)}$ \\
\hline $3916 \cdot 64$ & 5 & $D$ & $16 \cdot 70 \mathrm{Cl}$ II $(68)$ & $3966 \cdot 14$ & - & - & - \\
\hline $3917 \cdot 62$ & 2: & $B$ & $17 \cdot 57 \mathrm{Cl}$ II $(68)$ & 3967.09 & - & 一 & - \\
\hline $3917 \cdot 92$ & - & - & - & $3970 \cdot 08$ & 4770 & $A$ & $70.074 \mathrm{H}$ \\
\hline $3918 \cdot 54$ & - & - & - & $3973 \cdot 26$ & 20 & $A$ & $73 \cdot 263$ O II $(6)$ \\
\hline 3918.93 & 79 & $A$ & $\begin{array}{l}18 \cdot 977 \text { C II (4) } \\
19 \cdot 005 \text { N II }(17)\end{array}$ & & & & \\
\hline
\end{tabular}

the spectral regions covered by our plates, and these results will be given separately. The equivalent widths and profiles agree tolerably well with those published earlier, but the greater wealth of the present material and the more carefully checked photometric calibration make the newer values more trustworthy.

I have chosen $\gamma$ Pegasi for more detailed illustration, because it is the star, for which the observations are most plentiful, cover the greatest spectral range and have been more fully worked up. It has been subjected to detailed study because of the richness of its spectrum, in spite of the fact that it is a variable of the $\beta$ Canis Majoris type (McNamara, 1953). Observations by Struve, as well as our own spectrophotometric measurements, show no change in the shapes or total intensities of the absorption lines. 
Our present programme includes stars ranging from late O (10 Lacertae) to B5 ( $\alpha$ Sculptoris; $v$ Andromedae), but principal emphasis is being placed on objects between $\mathrm{B} 0$ and B3, inclusive. The Mount Wilson material has been supplemented by observations supplied us by the McDonald, Perkins, and Dominion Astrophysical

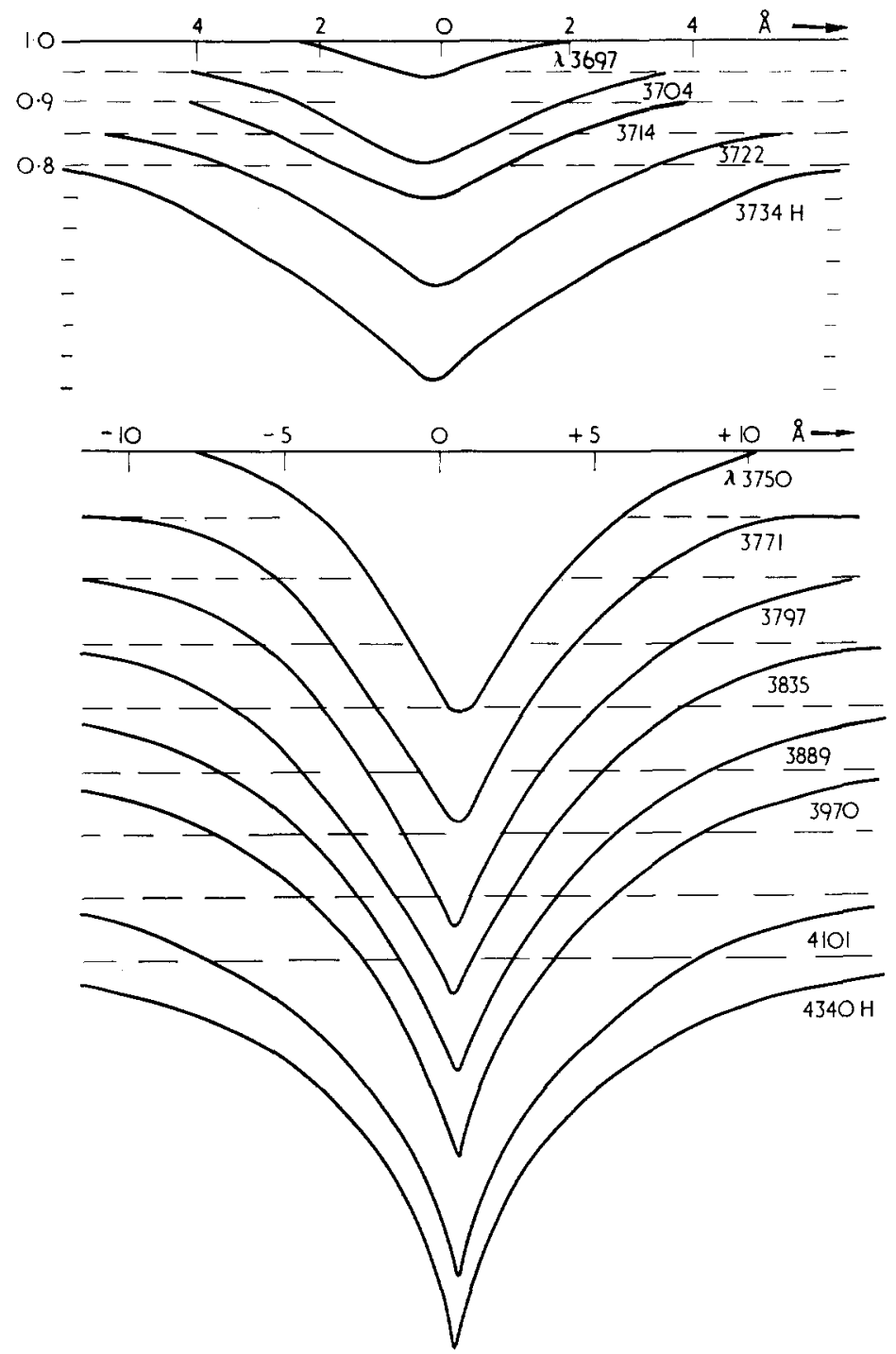

Fig. 4. Measured line profiles of hydrogen in $\gamma$ Pegasi

Observatories. The programme has been supported by a grant from the National Science Foundation.

\section{Curves of Growth for Early-type Stars}

A number of investigators have discussed the atmospheres of early-type stars with the aid of curves of growth (UNsöLd, 1941; ALLER, 1946). This powerful tool for a first reconnaisance of stellar atmospheres is based on the approximation that the spectral lines are formed in an isothermal atmosphere at constant pressure. In 

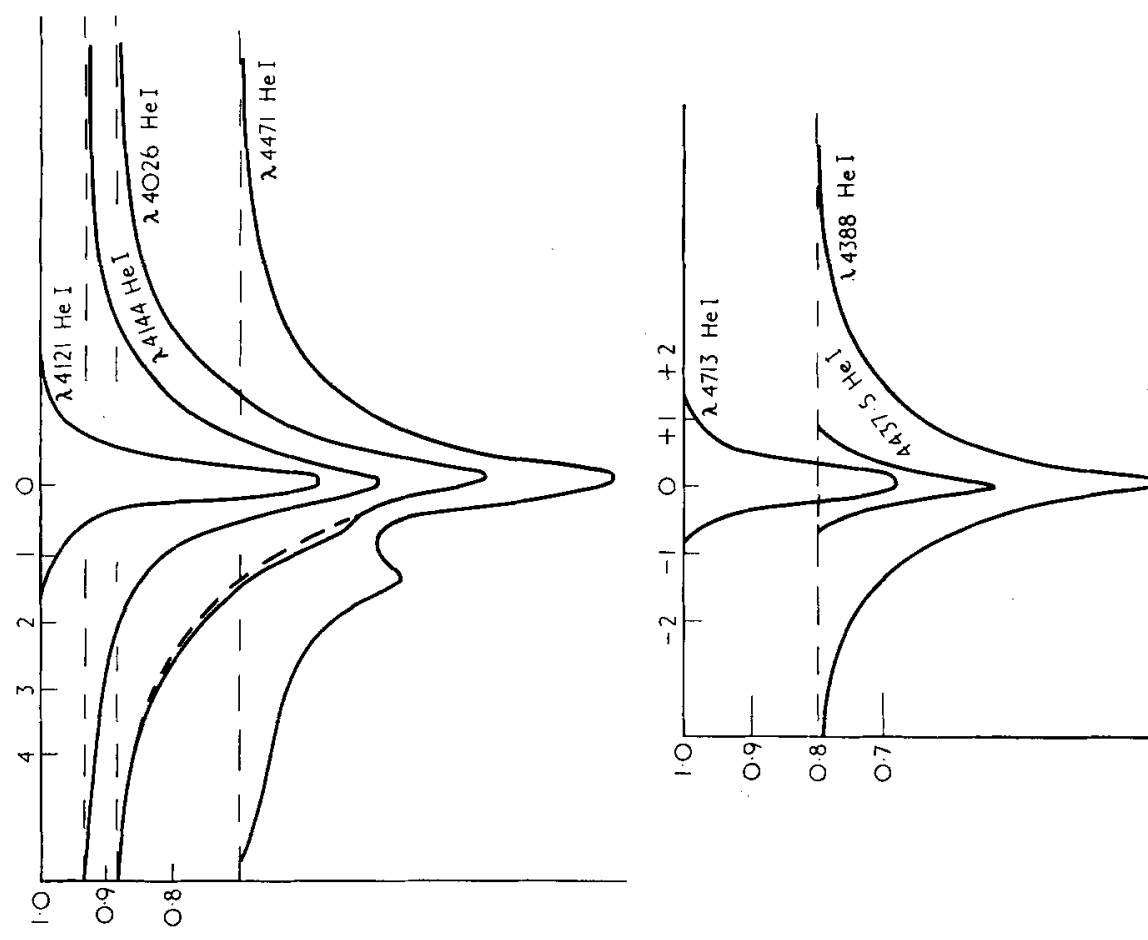

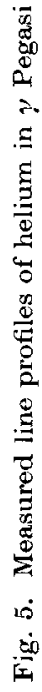

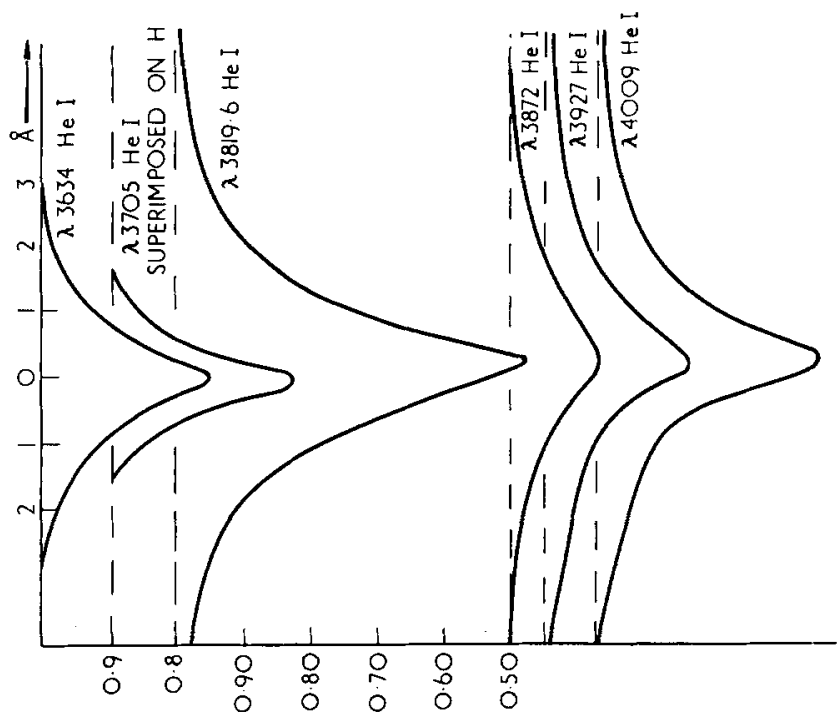


supergiant stars where no sound theoretical basis for the construction of model stellar atmospheres exists, the curve of growth procedure remains the most effective means for the analysis of the observations.

Fig. 6 shows the curve of growth for BIDELMas's helium star HD 160641 (BIDELMAN, 1952). The theoretical curve and the notation are those of UnsöLd (194I). The stronger lines appear to have a pronounced damping as a consequence of greater density in the line-forming layers than appears to exist in other stars of the same level of excitation. The chemical composition of the star appears to be about the same as

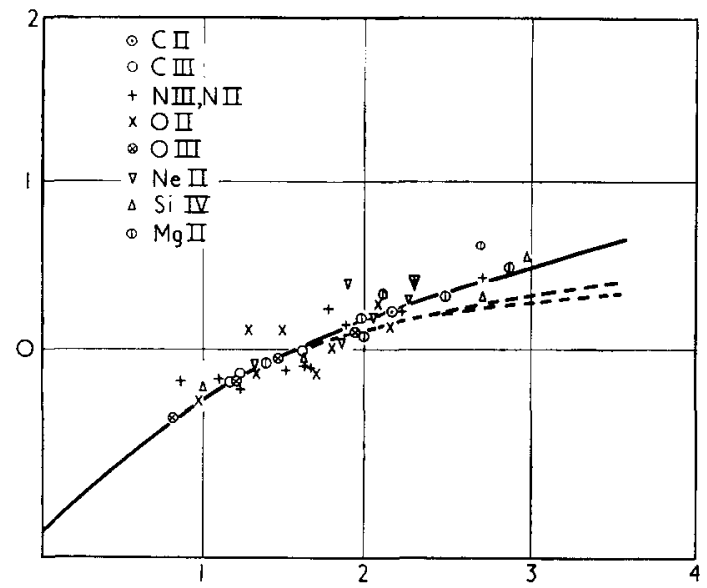

Fig. 6. Curve of growth for HD 160641

Ordinates are: $\log \frac{1}{R_{c}} \frac{W_{2}}{2 \Delta \lambda_{\mathrm{D}}}$; abscissae are: $\log \frac{1}{R_{c}} \frac{N H f}{\Delta w_{\mathrm{D}}}$. The notation is that of Unsöı. (1941). A turbulent velocity of $14 \mathrm{~km}$ per sec. and a temperature of $31,500^{\circ} \mathrm{K}$ are assumed

that of $\gamma$ Pegasi (Aller, 1949) or 10 Lacertae (Aller, 1946), but with the hydrogen replaced by helium.

The difficulty with the curve of growth analysis is that it assumes that all parts of all lines are formed at the same temperature and density. Obviously, such an approximation is very poor if one wishes to compare, for example, the Si II and Si IV lines in the spectrum of a star such as $\gamma$ Pegasi.

\section{Concerning Model Atmospheres}

Model atmospheres for high temperature stars have been calculated by many investigators: RudKJöBING, 1947; AlLER, 1949; UNDERHILL, 1950, 1951a; Pecker, 1950; Neven, 1952; Milligan and Aller, 1953, 1954; McDonald, 1954; SAITo, 1954. For such computations it is necessary to assume a chemical composition and to know the coefficient of continuous absorption as a function of frequency. The simplest models are those of pure hydrogen. Except for the contribution of the free-free transitions, reliable calculations of the coefficient of continuous absorption for hydrogen have been made (Menzel and PEKkrIs, 1935*).

The real difficulty in the calculation of model atmospheres lies in our ignorance of the exact relation between the temperature and the optical depth in the star. In the early B-type stars no convection occurs unless a considerable proportion of helium

* The equation (2.25) given by these authors does not hold at high temperatures. 
is present. That is, these atmospheres are in radiative equilibrium which implies that the flux is constant. Miss McDonald (1954) showed that this constancy of flux condition is a poor criterion for fixing the temperature distribution. The physical reasoning is easy to understand. The total flux at any point comprises contributions from many different strata embracing a large range in temperature. Hence, slight adjustments in the temperature distribution, which will have perceptible effects on the predicted line spectrum, may show no effect on the flux at all!

In the models calculated by MiLLIGAN and the writer $(1953,1954)$, a flux constant to within less than 1 per cent was obtained in four approximations. In these calculations we employed the optical depth at $\lambda 5000$ as the independent variable. We took into account the radiation pressure and tried to allow for the blanketing effect in accordance with a formula suggested by CHANDRASEKHaR (1936; see equation 52). The blocking of the outgoing radiation by the hydrogen lines and Lyman continuum produces a steep temperature gradient near the surface of the star. Beyond optical depths greater than about $0 \cdot 3$, the temperature increases relatively slowly.

Although the constancy of flux condition and the blanketing effect establish the expected trend of the temperature distribution, we must use the line spectrum to improve the latter. For example, in $\gamma$ Pegasi we could use the lines of Si II, Si III, and $\mathrm{Si}$ IV to establish the temperature distribution over a considerable range in optical depth.

The hydrogen line profiles could also be used to improve the structure of the model atmosphere, provided we have an exact theory of the line broadening coefficient. We return to this topic in Section 5.

The central intensities of the hydrogen lines do require a sharp temperature drop near the surface of the B stars. The existence of a marked blanketing effect is strikingly shown, for example, in $\alpha$ Sculptoris, B5, where the lines of Fe II and Ti II (which we usually associate with stars of spectral class A0 and later) are prominent. In the same spectrum are strong lines of helium, and lines of $\mathrm{N}$ II and $\mathrm{O}$ II which we produced in the deeper layers of the atmosphere.

Recent calculations by J. E. MILLIGan show that the STRömGREN-method weighting function for the evaluation of the flux may give errors as large as 10 per cent in the neighbourhood of the Balmer discontinuity. The effect of using the STRömGrEN approximations on the constancy of flux condition remains to be tested.

With a view to possible applications to helium stars such as HD 124448 (PoPPER, 1947) and HD 160641, as well as to certain of the very blue stars observed in globular clusters we have initiated the calculation of model atmospheres for pure helium stars. The coefficients of continuous absorption for neutral helium are not as accurately known as for hydrogen. The inaccuracies are particularly serious for the observable spectral regions. The theoretical difficulties are considerably greater than for pure hydrogen atmospheres since helium can exist in two stages of ionization. In a star with an effective temperature near $30,000^{\circ} \mathrm{K}$ most of the helium is singly ionized in the outermost layers which are in radiative equilibrium. With increasing depth in the star, helium becomes doubly ionized, and a transition to a convection zone occurs quickly. Within a short distance the energy transport switches from radiative transfer to convective transport. The final model can be reached only on the basis of successive approximations; one must consider, for example, the influence of the partition functions for neutral and ionized helium upon the degree of ionization. Nevertheless, the continuous energy distributions seem capable of more or less 
precise prediction. They differ appreciably from those computed for pure hydrogen atmospheres, there being no Balmer discontinuity, and would appear to give at least a qualitative explanation for some of the apparently abnormal colour relations found by H. C. ARP and HAROLD JoHnson in certain blue stars of globular clusters.

Observational checks of theoretical helium atmospheres are less easy to make because of the limited observational material at present available.

\section{Experimental Studies on Line Broadening}

The ultimate objective of the construction of model atmospheres is the calculation of actual abundances from the profiles and total intensities of spectral lines. Given the temperature and density distribution in the atmosphere and the mechanism of line formation, we can calculate the theoretical profile and total intensity of the line if we know the strength or $f$-value and the line broadening coefficient.

For most elements we can measure only the equivalent widths, but for the hydrogen and helium lines the profiles may be measured as well. Hence, the observed line shapes carry important clues to the structure of the atmosphere provided we can interpret them. Turbulence and rotation affect the shapes of all lines, whereas the interatomic linear Stark Effect influences only those of hydrogen and helium.

Fortunately, the broadening of the hydrogen lines can be studied in the laboratory under controlled conditions. An important part of our programme on the structure and chemical compositions of the atmospheres of early-type stars is the accurate measurement of the coefficients of line absorption for the Blamer lines with the aid of the luminous shock tube. A gas of low molecular weight such as hydrogen or helium is confined at a high density behind a membrane while the rest of the tube contains at low density a heavier gas such as xenon or argon with which a small amount of hydrogen is mixed. The membrane is ruptured, the dense gas expands as a faintly luminous shock wave rushes down the tube. When this shock wave strikes the wall at the far end of the tube and is reflected, the temperature of the gas is raised to values of the order of $10,000-20,000^{\circ} \mathrm{K}$, depending on the Mach number of the shock wave, and the gas becomes intensely luminous. Eugene Turner and Alan Kolb have made a careful quantitative study of this phenomenon with the aid of a spectrograph and a revolving drum camera. Their time-resolved spectra show that the hydrogen lines behind the reflected shock are broadened by an amount that can be accurately measured with a good spectrograph. Later, as two or more shock waves interact, the broadening increases but the lines finally narrow down as the excitation dies away.

The importance of the shock tube as a tool for astrophysical research lies in the circumstance that the shock-tube equations permit the calculation of the temperature and density as a function of position and time. It is possible to allow for the effects of excitation and ionization upon the temperature and density.

TURNER and KOLB found that the widths and shapes of the hydrogen lines did not agree with the predictions of the Holtsmark Theory. The work of Jüraens (1952) at Kiel, on the other hand, appeared to support the Holtsmark Theory. The Kiel experiments involved a stabilized whirling stream of water in a tube along whose axis an electric arc was struck. Hydrogen and oxygen lines are observed with great strength. The temperature and density are established from the character of the observed spectrum in the Kiel work, whereas in the shock tube work the temperature and pressure are found from hydrodynamical considerations. The most recent work 
at Kiel indicates that for certain domains of temperature and density, at least, the Holtsmark Theory does not hold.

KOLB (1954) has made a detailed study of the line broadening in hydrogen. The HoLTsmark (1919) statistical theory ignores the motions of the perturbing particles, a neglect which can be important at high temperatures. It is not valid near the line core (SPITzER, 1940), nor in the far wings which are sensitive to close encounters. For short-range interactions, the electric field becomes inhomogeneous. The theory over-estimates the effect of large electric fields caused by close encounters since it assumes that the ions are distributed completely at random (ODGERs, 1952; CHANDRASEKHAR, 1943). It neglects the broadening by electrons but KIVEL (1954) has shown that this is probably not a serious error, since the Holtsmark broadening by ions is about ten times larger than that by electrons at an ion density of $10^{14}$ per $\mathrm{cm}^{3}$.

Near the line core the more distant collisions become important and here the socalled collision damping theories are applicable (SPITzER, 1940). Because of the long range of the Coulomb forces in the first-order Stark Effect, it is necessary to include not just single encounters but the effects of two or more perturbing particles acting at once. For the region of the line core, and the first order Stark Effect, Koub finds the following expression for the intensity distribution in the spectral line

$$
A_{\kappa}((\omega))=\frac{2}{\pi} \alpha_{\kappa} \frac{\left(\omega-\omega_{0}\right)^{2}}{\left[\left(\omega-\omega_{0}\right)^{2}+\alpha_{\kappa}^{2}\right]^{2}}
$$

Here $\omega$ is the circular frequency, $2 \pi v$, while

$$
\alpha_{\kappa}=\frac{2 \pi N_{i}}{n-1}\left[\frac{\sqrt{\pi} \Gamma\left(\frac{n-1}{2}\right)}{\Gamma\left(\frac{n}{2}\right)} \frac{3 h}{4 \pi m} \cdot \frac{\chi_{\kappa}}{Z}\right]^{2 /(n-1)} \frac{F(n)}{\bar{v}^{(3-n) /(n-1)}}
$$

where $N_{i}$ is the ionic density, $m$ the mass of the electron, $h=$ Planck's constant, $Z=1$ for hydrogen and 2 for ionized helium. Here $\chi_{\kappa}$ refers to particular pairs of Stark components as computed for $\mathrm{H} \gamma$, for example, by Miss UNDERHILL (1951b). The $\bar{v}$ refers to the velocity of the perturber, $n$ is the exponent in the force law, while

$$
F^{\prime}(n)=\int_{0}^{\infty} \frac{d \chi}{\chi^{n+1 / n-1}}\left(1 \ldots \frac{\sin \chi}{\chi}\right)
$$

is an integral which has been tabulated by ChandRasekhaR (1948). $F(n)$ diverges for $n \leq 2$, but KoIB and TURNER find an effective value of $n$ somewhat greater than 2 . That is, for the first-order Stark Effect, the force law varies not as $r^{-2}$ but at a slightly steeper rate because the contribution of the more remote and oppositely charged electrons and ions tend to cancel out.

A comparison of $A_{\kappa}(\omega)$ and Holtsmark's $W(\beta)^{*}$, with both functions normalized shows that the former is shallower in the core and more extended in the wings than is the latter. Furthermore, the frequency distribution in the core of $\mathrm{H} \gamma$ and the size of the splitting of $\mathbf{H} \beta$ are explained to within the experimental error.

* The function $W(\beta)$ is tabulated by VerwhiJ (1936). 
The shapes of the line absorption coefficients can be determined throughout the entire frequency range of the line by experimental methods. LowELL DoherTy, in collaboration with TURNER and KOLB, is carrying out such measures for the principal lines of the Balmer series over a range of temperature and density of astrophysical interest.

KoLB has pointed out that the confluence of the Balmer lines will depend on line broadening effects operating near the line core-not in the wings. Hence the INGLIS-Teller (1940) formula will have to be modified. The numerical constant will depend on $F(n)$ which must be established empirically, and the resultant formula for the ion density will involve the temperature.

Detailed applications of the shock-tube results to the calculation of the profiles of $\mathrm{H} \alpha$ and other Balmer lines must await improved experimental data and accurate line profiles. With the new line broadening data, the hydrogen lines can then be used as tools for the improvement of model stellar atmospheres, especially for introducing the small corrections to the temperature distribution that are necessary for the interpretation of the line intensities. Unfortunately, the broadening of the helium lines cannot yet be studied in our laboratory, although appropriate experiments may be devised eventually.

At the moment, it is not possible to answer such questions as the hydrogen/ helium ratios in the hotter stars since the result will depend critically upon the broadening mechanisms and upon the structure of the atmosphere. If the experimental possibilities are carefully exploited it will be possible to obtain data, not only on the line broadening, but also on $f$-values for atoms such as argon or neon as well. The stars are so complex that we cannot afford to neglect any experimental technique that promises to provide us with necessary basic physical data.

To summarize, our programme on the analysis of the hotter stars consists, on the one hand, of the assemblage of the best observational data on the line profiles and intensities, and on the other, of the interpretation of these data with the best theoretical methods we can devise, aided whenever possible* by direct experimental work.

I am grateful to Messrs. Kolb and Turner who communicated their results to me in advance of publication. I secured the observational data in 1952 while working as a guest investigator at the Mt. Wilson Observatory. Mr. James MrLligan made the microphotometer tracings. Special thanks are due to Mr. JUN JUGAKU for his assistance in the preparation of Table 1 and the measurement of the line profiles on the tracings.

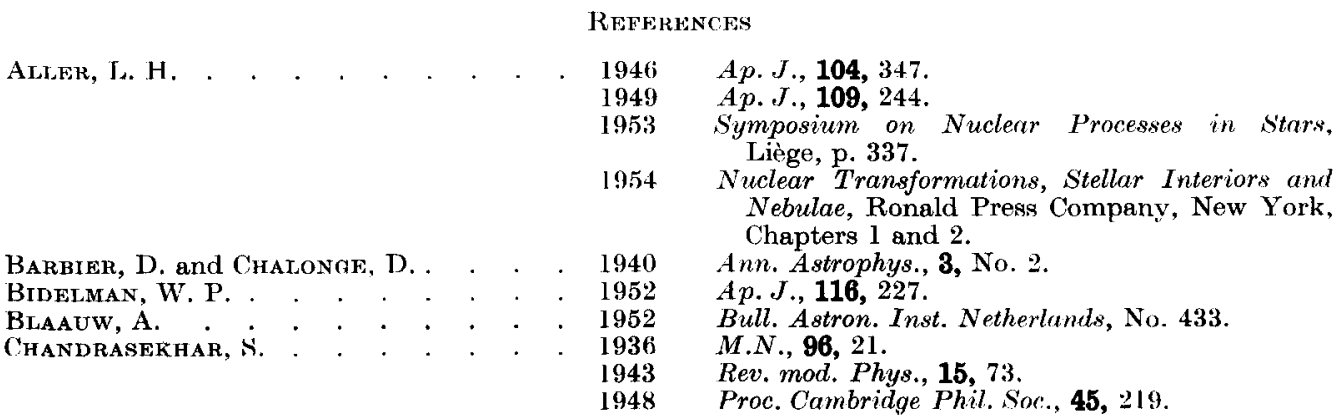

- A description of some aspects of the observational end of the progranume is given in 1. H. ALLER (1953). 
Code, A. and Whitford, A. E. . . . . 1954

HoltsmaRK, J.. . . . . . . . . 1919

Inglis, D. R. and Teller, E. . . . . 1940

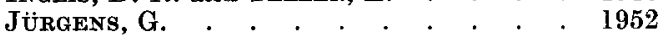

Kienle, H., Wempe, J. and Beileke . . 1940

KIVEL, B. . . . . . . . . . . . 1954

Kоцв, А. C. . . . . . . . . . . $\quad . \quad 1954$

McDonald, J. K. . . . . . . . . 1954

MoNamara, D. . . . . . . . . . . 1953

Menzel, D. H. and Pekeris, C. L. . . . 1935

Milligan, J. E. and Allwr, L. H. . . . 1953

1954

MoOre, C. E. . . . . . . . . . . . 1945

Neven, L. . . . . . . . . . . . . . . 1952

ODAERs, G. L. . $\quad . \quad . \quad . \quad . \quad . \quad . \quad . \quad . \quad . \quad 1952$

PECKER, J. C. . . . . . . . . . . . . . 1950

POPPER, D. M. . . . . . . . . . . . . . . . 1947

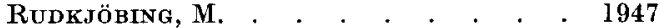

SaIto, S. . . . . . . . . . . 1954

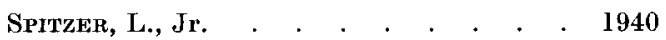

UNDERHILL, A. B. . . . . . . . . 1948a

$1948 \mathrm{~b}$

1950

1951 a

$1951 \mathrm{~b}$

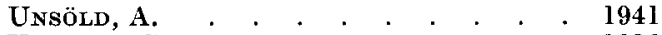

VERWFIJ, S. • • . . . . . . . . . 1936

WILLIAMS, R. C. . . . . . . . . 1938
Unpublished.

Ann. Phys. (Leipzig), 58, 576.

Ap. J., 90, 439.

Z. Phys., 134, 21.

Z. Astrophys., 20, 91.

Thesis, Yale University.

Conference on Stellar Atmospheres, Ed. by W. WRUBEL, Indiana University, Bloomington, Indiana.

Publ. Dominion Astrophys. Obs., 9, 269, 1953.

Publ. A.S.P., 65, 144.

M.N., 96, 77 .

Astron. J., 58, 45.

Conference on Stellar Atmospheres, Indiana University.

Princeton Obs. Contrib., No. 20.

Dissertation, Liège.

Ap. J., 116, 444 .

Ann. Astrophys., 13, 294, 319, 433.

Publ. Astron. Soc. Pacific, 59, 320.

Publ. Copenhagen Obs., No. 145.

Contrib. Kyoto University Institute of Astrophysics, No. 48.

Phys. Rev., 58, 348.

Ap. J., 10\%, 337 .

Ap. J., 10\%, 349.

Publ. Copenhagen Obs., No. 151.

Publ. Dominion Astrophys. Obs., 8, 357.

Publ. Dominion Astrophys. Obs., 8, 385.

Z. Astrophys., 21, 1.

Publ. Astron. Inst. Amsterdam, 5.

Publ. Obs, Michigan, 7, 93.

Publ. Obs. Michigan, $7,147,159$.

\title{
The Technique and Possibilities of Astronomical Spectrophotometry
}

\author{
Jesse L. Greenstein \\ Mount Wilson and Palomar Observatories, \\ Carnegie Institution of Washington, \\ California Institute of Technology, \\ Pasadena, California, U.S.A.
}

\section{SUMmaRY}

The present capabilities of photographic spectrophotometry are reviewed; with large reflectors and modern optics accurate work on all naked-eye stars is possible. Photo-electric spectral scanning is best suited for low-resolution; image intensification will be needed to avoid losses due to scanning. The technique for theoretical analysis of spectra for the abundances of the elements is briefly discussed. The ability to observe very faint stars with moderately high dispersion at Palomar has opened several new fields of research, especially in the study of the white dwarfs. New results include line profiles as affected by Stark and pressure broadening, and the existence of shells of low-pressure gas around several helium-rich and low-temperature white dwarfs.

THE wavelengths and intensities of spectral lines provide us with a major part of our information concerning the nature of the stellar universe. Precise wavelengths, with accuracies of one part in a million, are readily obtainable. Line profiles, and equivalent widths, however, are seldom determined with an accuracy of even 5 per cent. Photography of a spectrum offers the enormous advantage of simultaneous accurate recording of the positions of the lines. The complex and very non-linear 